\title{
Review of: "Survival of Campylobacter jejuni in Amoebae enhances subsequent invasion of mammalian cells"
}

\author{
Maja Šikić Pogačar ${ }^{1}$ \\ 1 University of Maribor
}

Potential competing interests: The author(s) declared that no potential competing interests exist.

I read the manuscript with pleasure!

It is an interesting study that used amoebae as a transient host for $\mathrm{C}$. jejuni and later investigated the invasion potential of those bacteria for re-infection of amoeba, two cell lines and also Galleria mellonella compared to control C. jejuni that were not incubated with amoeba.

The manuscript is well written however, I have some suggestions to improve the readability of the manuscript.

\section{Abstract:}

Line 33-35: It is difficult to understand the sentence. It would be easier if you would put also 11168H CBA into parenthesis as you did with the other two. Please mention also cell lines in the parenthesis.

Introduction:

Line 53: Being is not correct tense. remove it

Line 70: What do you mean with increased disease in mammalian hosts? Do you mean more severe disease or such bacteria might be capable of causing more cases of disease? Please rewrite for better clarity.

C. jejuni invasion and survival assay

Line 88: Why did you choose strain $11168 \mathrm{H}$ and not NCTC 11168 ?

Line 99: write bacterial cells or remove cells.

Line 102-104: You write that for invasion assay, the experiment was performed as described above, with additional step of serial dilutions etc.. But what for is used the procedure from line 95-102? Isnt that used for determining the invaded bacteria? Can you write it more clear?

Line 106: Can you write in short here which strain is $116 \mathrm{H}$ GFP even though it is mentioned in table 1. 
Line 110: please indicate time points here in parenthesis

Discussion is clear and well written and so is conclusion.

The literature used is up to date.

I suggest authors to do the corrections of the manuscript for better understanding and readibility. 